\title{
Preliminary Study on Unsupervised Classification of Remotely Sensed Images on the Grid
}

\author{
Jianqin Wang ${ }^{1}$, Xiaosong Sun ${ }^{1}$, Yong Xue ${ }^{1,2 *}$, Yincui $\mathrm{Hu}^{1}$, Ying Luo ${ }^{1}$, \\ Yanguang Wang ${ }^{1}$, Shaobo Zhong ${ }^{1}$, Aijun Zhang ${ }^{1}$, Jiakui Tang ${ }^{1}$, and Guoyin Cai ${ }^{1}$ \\ ${ }^{1}$ Laboratory of Remote Sensing Information Sciences, Institute of Remote Sensing \\ Applications, Chinese Academy of Sciences, P. Box 9718, Beijing 100101, China \\ ${ }^{2}$ Department of Computing, London Metropolitan University, 166-220 Holloway Road, \\ London N7 8DB, UK \\ \{tian1.wang@163.com,y.xue\}@londonmet.ac.uk
}

\begin{abstract}
Grid is a new technology. With corresponding middleware it can give strong computing power. In this paper we mainly discuss the middleware technology and architecture used in remote sensing image classification algorithm. Because unsupervised classification middleware is the key of the classification middleware algorithms, we study the alternant-unsupervised middleware and put forward a non-alternant unsupervised middleware scheme. Based on this scheme, main factors which effect the performance of nonalternant unsupervised classification are analyzed.
\end{abstract}

\section{Introduction}

"Grid" computing has emerged as an important new field, distinguished from conventional distributed computing by its focus on large-scale resource sharing, innovative applications, and, in some cases, high-performance orientation [1]. They defined "Grid problem", which is defined as flexible, secure, coordinated resource sharing among dynamic collections of individuals, institutions, and resources -what they referred to as virtual organizations(VO).

The term "the Grid" was coined in the mid 1990s to denote a proposed distributed computing infrastructure for advanced science and engineering [2]. There are several famous grid projects today. Access Grid(ww.fp.mcs.anl.gov/fl/access grid) lunched in 1999 and mainly focused on lecture and meetings-among scientists at facilities around the world. European Data Grid sponsored by European union, mainly in data analysis in high-energy physics, environmental science and bioinformatics. Grid Physics Network (GriDPhyN) [5] lunched in 2000 and sponsored by NSF mainly in data analysis for four physics projects: two particle etectors at CERN's Large Hadron Collider, the Laser Interferometer Gravitational Wave Observatory, and the Sloan Digital Sky Survey. Information Power Grid [6] is the NASA's computational support for aerospace development, planetary science and other NASA research. International Virtual DataGrid Laboratory (iVDGL)[7] sponsored by NSF and counterparts in Europe, Australia, and Japan in 2002. Network for Earthquake Engineering and Simulation labs (NEESgrid) (www.neesgrid.org) intended to integrate

\footnotetext{
* Corresponding author
} 
computing environment for 20 earthquake engineering labs. TeraGrid (www.teragrid.org) is the general-purpose infrastructure for U.S. science: will link four sites at 40 gigabits per second and compute at up to 13.6 teraflops. U.K. National Grid [8] sponsored by U.K Office of Science and Technology. Unicore (www.unicore.de) is a seamless interface to high-performance Education and Research computer centers at nine government, industry and academic labs

The famous Grid focused on spatial information includes SpaceGrid, EnvirGrid and EarthObsevation Grid. ESA's SpaceGrid is an ESA funded initiative (http: / / sci2.esa. int/spacegrid).

EnvirGrid main goals are generalization of Earth Science application infrastructure to become GRID-aware, extend GRID access to European Environmental and Earth Science application to large science communities, to value adding and commercial communities, ..., and demonstrate collaborative environment for Earth Science. Dozens of satellites constantly collecting data about our planetary system 24 hours a day and 365 days a year. Large scale of satellite data needed to be processed and stored in real time or almost real time. Real time processing in remote sensing is very significant in some cases, such as fire and flood. So far real time processing in remote sensing confronts much difficulties in one single computer, or even impossibility. Computing grid which is integrated by series of middlewares provide a way to solve this problem [3]. The following is our preliminary study on unsupervised classification middleware on the grid platform.

\section{Middleware on Unsupervised Classification on Grid Platform}

Classification is a important field in digital remote sensing image processing. Almost all classification algorithms involve processing pixel value one by one. Large amount of remote sensing data classification will take too long time. Luckily, emerging grid technology can provide large computing power in the Internet as the security type. The grid computing nodes resource distribute loosely. They may be supercomputers or common PCs with heterogeneous architecture. The grid computing nodes resource distribute loosely. They may be supercomputers or common PCs with heterogeneous architecture. Classification middlewares on grid platform can solve this problem.

\subsection{Testbed of Telegeoprocessing Grid Platform}

Telegeoprocessing grid platform in Institute of Remote Sensing Application, Chinese Academy of Science is an advanced High-Throughput Computing grid using condor which copyright is Computer Sciences Department, University of WisconsinMadison. Heterogeneous computing nodes including two sets of Linux computers and WINNT 2000 professional computers and one set of WINNT XP computer provide stable computing power. The grid pool uses java universe to screen heterogeneous characters. It is a fundamental bare computing platform and can not run remote sensing image processing program. So remote sensing image processing middlewares running on grid pool are inevitable. Common users can use the heterogeneous grid 
and share its strong computing power to process remote sensing images with middlewares as if in one supercomputer.

\begin{tabular}{|c|l|l|l|}
\hline IP & Name & Arch-OS & Role \\
\hline 192.168 .0 .5 & Manager.linux & Itel/Linux & Manager/client \\
\hline 192.168 .0 .3 & Wjq.linux & Intel/WIN50 & Client \\
\hline 192.168 .0 .102 & Tele1.linux & Intel/WIN50 & Client \\
\hline 192.168 .0 .111 & Tele2.linux & Intel/WIN51 & Client \\
\hline 192.168 .0 .6 & Client2.linux & Intel/Linux & Client \\
\hline
\end{tabular}

\subsection{A Simple Unsupervised Classification Algorithm Description}

The aim of the classification middleware on grid is to divide jobs into several assignments and submit them to the computing pool. The rule of dividing jobs is: the more simple the dividing type can be used, the better it is. Difference among all the heterogeneous computing nodes resource determine this rule. With the same algorithm, the amount of the remote sensing image data is the most significant one of all the factors of impacting the classification speed. Naturally, dividing jobs can be transferred into dividing data and sending data to several grid nodes properly

Training areas can be chosen beforehand in the supervised classification algorithm. Then interoperation among the divided areas doesn't exist at all. Dividing assignment is relevantly easy. Contrarily, There exists strong correlation among all the divided data parts in the unsupervised classification. Mean center of classification must change dynamically according to computing result of every time's cycle. So the key technology of classification middlewares is to solve unsupervised classification middleware.

Dynamical clustering method is a typical algorithm of all the unsupervised classification algorithms. Every pixel can be classified into corresponding $\mathrm{K}$ class group individually according to least -squares rule. The algorithm iterates over three steps:

1. Compute the mean of each cluster.

2. Compute the distance of each point from each cluster by computing its distance from the corresponding cluster mean. Assign each point to the cluster it is nearest to.

3. Iterate over the above two steps till the sum of squared within group errors cannot be lowered any more. Vector $\mathrm{x}$ is the pixel value. The $\mathrm{K}$-means algorithm partitions data into $\mathrm{K}$ clusters. The solution is then a set of $\mathrm{K}$ centers, each of which is located at the centroid of the data for which it is the closest center. For a partition $\mathrm{P}$ of the elements in $\{1,2 \ldots \mathrm{m}\}$, denote by $\mathrm{P}$ (i) the cluster assigned to $\mathrm{i}$ and $\mathrm{C}(\mathrm{j})$ the centroid of cluster $\mathrm{j}$. The intent of the K-means algorithm is the minimization of the objective function

$$
E p(i)=\sum_{i=1}^{m} \operatorname{dist}(i, C(P(i)))
$$


Where $\operatorname{dist}(x, c)$ is the distance between the vectors $\mathrm{x}$ and $\mathrm{c}$. In this experiment, we define the distance as the Euclidean distance

$$
\operatorname{dist}(x, c)=\|x, c\|
$$

\subsection{Alternant Unsupervised Classification Middlewares Test on the Telegeoprocessing Grid Testbed}

\section{Test Data Resource and Ground}

We employ IKONOS satellite dada as remote sensing image resource. IKONOS satellite images were acquired on 26 April 2002 with approximately $1 \mathrm{~m}$ and $4 \mathrm{~m}$ spatial resolution in the visible (panchromatic) and relatively broad bands in the blue, green, red, and near-infrared portions of the spectrum, respectively. We use bands of blue, green, red, and near-infrared for about $4 \mathrm{~m}$ spatial resolution. Test ground surrounds Olympic park in Beijing.

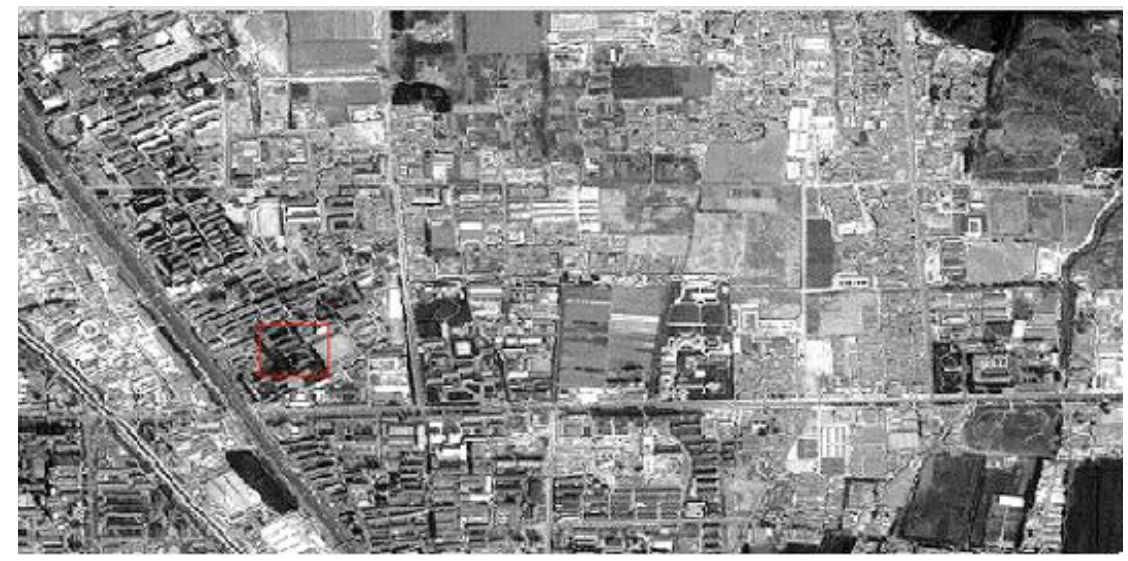

Fig. 1. The test area showed by IKONOS satellite blue band

\section{Architecture of Alternant Unsupervised Classification Middleware on Grid Testbed}

Alternant middleware can provide friendly interface to users. Figure 2 shows the architecture of alternant unsupervised classification middleware development.

User just expresses the number of jobs, which he wants to divide the job into through the user interface. JDMM that denotes job dividing manager module can divide job according to the user's demand. JSMM (Job submission manage module) can submit jobs to grid platform. CJMM is circulating job monitoring manage module the duty of which is monitoring when every job's result can be sent back for each loop. It also computes the new mean center through RRM, which receives result. 
CJMM sends message to JSMM when the classification precision is not compatible to the user's demand. DFVM is data fusion and visualization module. It can fuse the divided result and give user visualization of the classification.

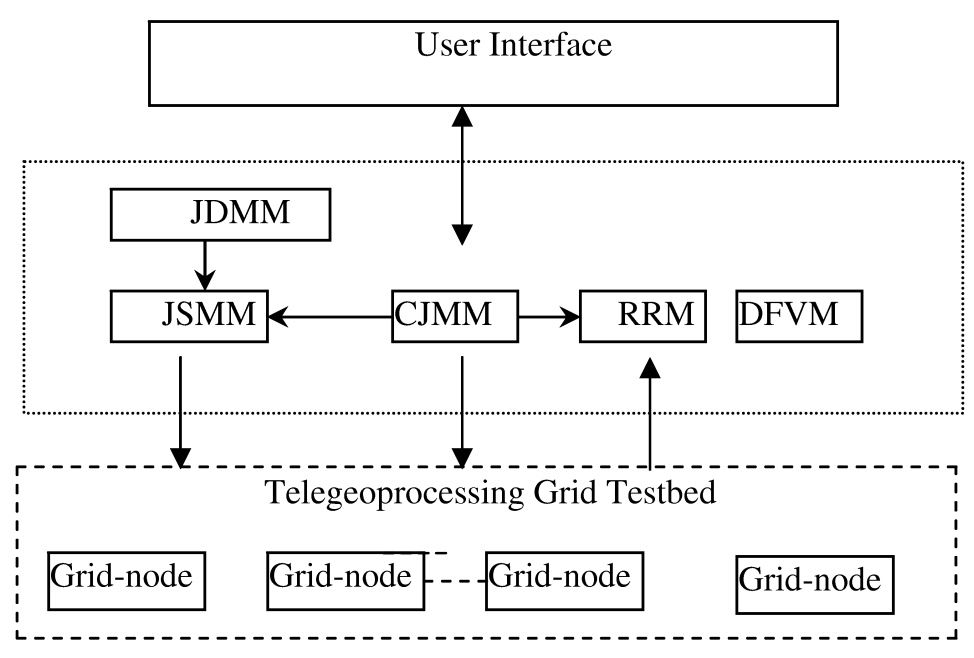

Fig. 2. Architecture of alternant unsupervised classification middleware

\subsection{Non-alternant Unsupervised Classification Middleware Study}

Alternant unsupervised classification middleware is natural to come to our minds. But alternant algorithm must employs result from every grid node of each loop. Thus job's submission of this time must wait for result of the last time. Frequent communication among grid nodes prolongs computing time. All the computing nodes may be heterogeneous and super-performed nodes must wait for common-performed nodes. Then the total time can be expressed as following:

$$
T_{\text {total }}=n * \max \left\{T_{\text {grid_node }}\right\}+n * T_{\text {communication }}
$$

where:

$\mathrm{n}$ : times of loop;

$\mathrm{T}_{\text {grid_node: }}$ computing time of one grid node for each loop;

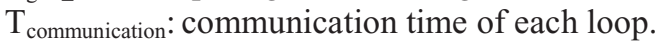

It is obvious that alternant unsupervised middleware algorithm is not the ideal one. So non-alternant unsupervised middleware is expected. We image that each job partition data should be computed individually and be submitted only once. After computing they can be fused and displayed. Through deep analysis we find that we can try to solve this problem for several reasons. Object can be distinguished and classified whatever in one whole image or in divided parts according to brightness of every 
pixel. The most important issue we care about is which type every object can be classified and the difference among different objects. We can do not care about the brightness of the whole core center. We set the classified number of each divided part. The problem may be that each divided part must be classified into given class number but it may be less the number in just one divided part. Through amalgamating dynamically class number through critical threshold we can adjust the class number in each divided part. Then classifying effect in each part can be almost the same as in one whole image after properly matching colors.

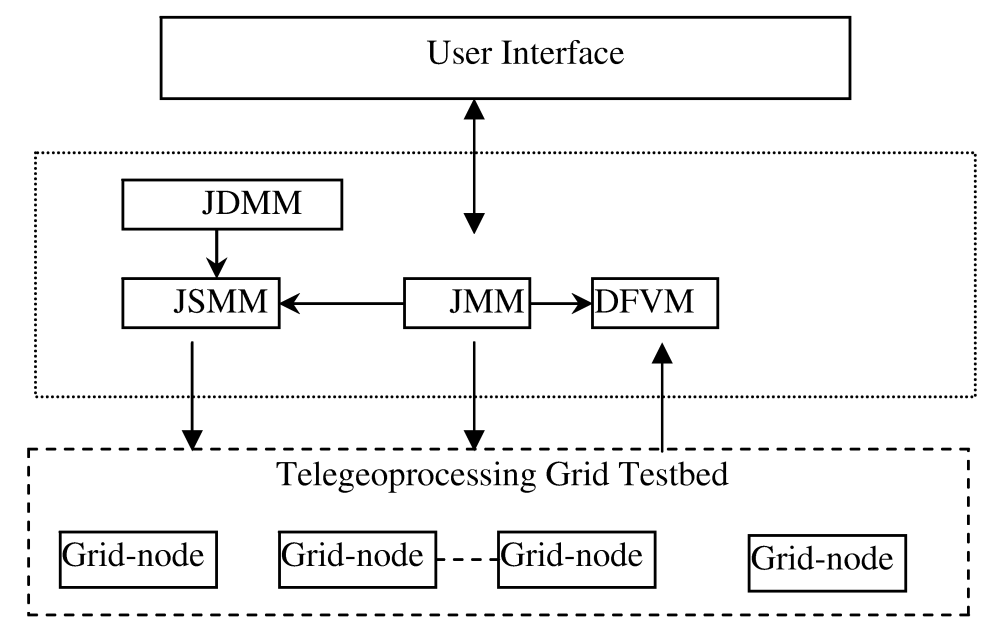

Fig. 3. Architecture of non-alternant unsupervised classification middleware

The assignment can be submitted only once. DFVM (data fusion and visualization module) gives users classification result when JMM (job monitoring module) finding the end result. The following pictures are the classification result comparison between single computer and non-alternant in grid pool. The effect shows almost little difference and approve we can employ this way to exploit our non-alternant middleware in grid environment. To distinguish vegetations from other objects we employ NDVI algorithm $(>=0.281)$ [4].

\subsection{Performance Test Using Non-alternant Middleware on Grid Platform}

Non-alternant middleware has advanced performance relative to alternant one. Multicomputing nodes in grid environment can give strong computing power. But the idea that the lesser time the assignment can take, the more job will be divided to is not always true. To find out which factor affect the performance most we have several test on this grid platform. Using non-alternant classification middleware we divide assignment into 4 and 16 job units individually. Test result is shown in the following table: 


\begin{tabular}{|c|c|c|}
\hline $\begin{array}{c}\text { Number of jobs } \\
\text { divided }\end{array}$ & $\begin{array}{c}\text { Time (full resource) } \\
\text { Second }\end{array}$ & $\begin{array}{c}\text { Time (partial resource) } \\
\text { Second }\end{array}$ \\
\hline 1 & 35 & 28 \\
\hline 4 & 25 & 41 \\
\hline 16 & 39 & \\
\hline
\end{tabular}

When all the grid nodes are free, assignment divided into 4 sub-jobs takes about 25 seconds and less than on single computer. But the same assignment divided into 16 sub-jobs takes longer time on the contrary for about 39 seconds. While computers in grid nodes are partially used, the time the same assignment takes will be longer than the free station.

Relationship between the number of the sub-jobs and the number of the computing nodes directly infects the performance of non-alternant classification middleware. When the number of the sub-jobs is almost the same as that of the computing nodes the performance of the middleware will be good. But when the number of the subjobs is far more than that of the computing nodes all the sub-jobs will wait in the working queues and the performance will fall.

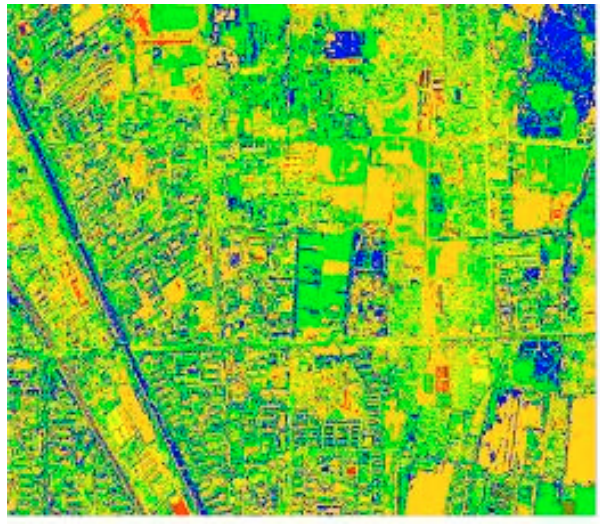

Fig. 4. Classification result on single computer.

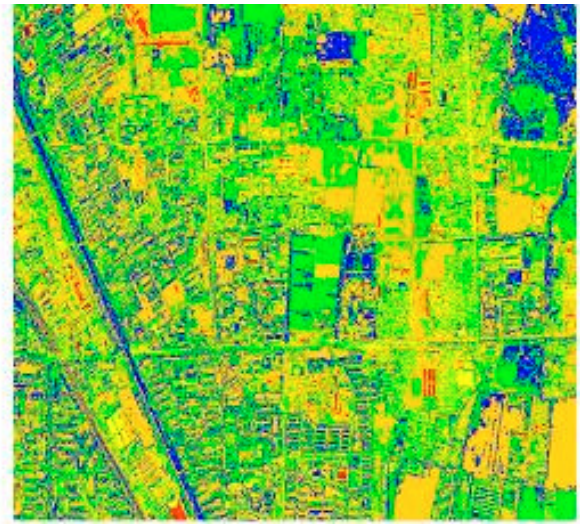

Fig. 5. Classification on Grid platform

\section{Conclusions}

Grid technology is very effective method for remote sensing image classification. It can give strong computing power in grid environment. Unsupervised classification middleware is the key technology of classification algorithms because of its alternant character. Developing non-alternant middleware of unsupervised classification can successfully use grid to classify remote sensing image objects. It is a good experience for other middleware used in remote sensing image processing. The number of divided sub-jobs must be proper according the actual station of the grid resource. If the number of divided sub-jobs is too more, the performance will be fall on the contrary in the special grid environment. 
Acknowledgement. This publication is an output from the research projects "CAS Hundred Talents Program", "Digital Earth" (KZCX2-312) funded by Chinese Academy of Sciences and "Dynamic Monitoring of Beijing Olympic Environment Using Remote Sensing" (2002BA904B07-2) funded by the Ministry of Science and Technology, China.

\section{References}

1. I. Foster, C. Kesselman, S. Tuecke The Anatomy of the Grid, Intl J. Supercomputer Applications, 2001.

2. Foster, I. and Kesselman, C. (eds.). The Grid: Blueprint for a New Computing Infrastructure. Morgan Kaufmann, 1999.

3. Jianqin Wang, Yong Xue, and Huadong Guo, 2003, A Spatial Information Grid Supported Prototype Telegeoprocessing System. In Proceedings of 2003 IEEE International Geoscience and Remote Sensing Symposium (IGARSS'2003) held in Toulouse, France on 21-25 July 2003.

4. F. Hirota, M. Morisugi, F. Weihua, and H. Imura: "A study on evaluations for vegetation in urban area with GIS and high-resolution satellite data - Reexamination of method to extract vegetation zones with the precedent satellite data -", Proceedings of 29th Annual meeting of Environmental Systems Research 2001, pp. 163 - 168, 2001.

5. Grid Physics Network. http://www.griphyn.org/index.php

6. W. E. Johnston, IPG Chief Architect. Information Power Grid. http: / / www.ipg.nasa.gov/

7. IVDGL Grid. http://www.ivdgl.org/

8. UK e-Science Grid Support Center. http://www.grid-support.ac.uk/ 